\title{
Bendless modulates JNK-mediated cell death and migration in Drosophila
}

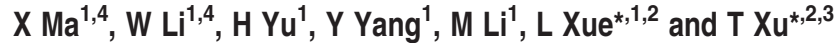

The TNF-JNK pathway is a highly conserved signaling pathway that regulates a wide spectrum of biological processes including cell death and migration. To further delineate this pathway, we carried out a genetic screen for dominant modifiers of the cell death phenotype triggered by ectopic expression of Eiger (Egr), the Drosophila TNF ortholog. Here we show that Bendless (Ben), an E2 ubiquitin-conjugating enzyme, modulates Egr-induced JNK activation and cell death through dTRAF2. Furthermore, Ben physically interacts with dTRAF2 and regulates Egr-induced dTRAF2 polyubiquitination. Finally, Ben is required for JNKdependent tumor progression, cell migration, oxidative stress resistance and longevity. Our results indicate that Ben constitutes an essential component of the evolutionarily conserved TNF-JNK pathway that modulates cell death and invasion, tumor progression, stress response and lifespan in metazoans.

Cell Death and Differentiation (2014) 21, 407-415; doi:10.1038/cdd.2013.154; published online 25 October 2013

Tumor necrosis factor (TNF) is an important cytokine that regulates a variety of cellular processes including proliferation, differentiation and survival, and has been implicated in the pathogenesis of many diseases including cancer, insulin resistance and autoimmune disorders. ${ }^{1}$ The $\mathrm{c}$-Jun $\mathrm{N}$-terminal kinase (JNK) is a key downstream mediator that, upon activation through the MAPK cascade, translocates to the nucleus where it phosphorylates and activates the transcription factors including AP-1. ${ }^{2}$ This signaling pathway has been conserved in Drosophila melanogaster, in which the TNF ortholog Eiger (Egr) triggers the activation of Basket (Bsk), the Drosophila JNK, through the JNK kinase kinase dTAK1 and the JNK kinase Hemipterous (Hep). ${ }^{3,4}$

The Egr-JNK signaling pathway was originally reported as a crucial regulator of cell death in Drosophila development. ${ }^{3,4}$ Further studies suggested that JNK signaling also has a vital role in modulating stress resistance and tumor longevity. Recently, JNK signaling was shown to be upregulated by the loss of tumor suppressor genes, for example, scrib and csk, and be critically required for $\operatorname{Ras}^{V 12} / \mathrm{scrib}^{-/-}$-triggered tumor growth and metastasis, as well as loss of scrib or csk induced invasive cell migration. $^{7-13}$

Drosophila genetics has provided a powerful tool for dissecting conserved signaling pathways and their roles in development. Several novel components of the Egr-JNK signaling pathway, including Wengen (Wgn) and dTAB2, the Drosophila ortholog of TNFR and TAB2/3, respectively, have been isolated as dominant suppressor of Egr-triggered cell death. ${ }^{14,15}$ Our previous work has shown that the TNF receptor-associated factor dTRAF2, which encodes an E3 ubiquitin ligase, regulates JNK-mediated tumor progression, cell death, oxidative stress resistance and longevity in Drosophila. ${ }^{6,7}$ However, the E2 ubiquitin-conjugating enzyme that modulates dTRAF2 activity in this pathway has remained unknown.

bendless (ben) encodes an E2 ubiquitin-conjugating enzyme that has important roles in escape response, ${ }^{16}$ axon guidance ${ }^{17,18}$ synaptic growth and maturation, ${ }^{19}$ long-term memory, ${ }^{20}$ innate immunity ${ }^{21}$ and genomic integrity. ${ }^{22}$ In this report, we performed a genetic screen and identified Ben as a novel component of the Egr-JNK pathway. Our genetic and biochemical analysis further indicated that Ben acts as the E2 enzyme for dTRAF2 that regulates JNK signaling-mediated tumor growth and invasion, cell death, oxidative stress resistance and longevity in Drosophila.

\section{Results and Discussion}

Loss of ben suppressed ectopic egr-induced cell death. To identify additional components of the TNF-JNK signaling pathway in Drosophila, we performed a deficiency screen to search for dominant modifiers of the GMR $>$ Egr small eye phenotype (Figure $1 b)^{23,24}$ that results from ectopic Egr-induced JNK-mediated cell death.,4 Deletions

\footnotetext{
1Department of Interventional Radiology, Shanghai 10th People's Hospital, Shanghai Key Laboratory of Signaling and Disease Research, School of Life Science and Technology, Tongji University, Shanghai, China; ${ }^{2}$ Howard Hughes Medical Institute, Department of Genetics, Yale University School of Medicine, New Haven, CT 06519, USA and ${ }^{3}$ Institute of Developmental Biology and Molecular Medicine, Fudan-Yale Center for Biomedical Research, School of Life Sciences, Fudan University, Shanghai, China

*Corresponding author: L Xue, School of Life Science and Technology, Tongji University, 1239 Siping Road, Shanghai 200092, China.Tel: +86 21 65985407; Fax: +86 21 65985407; E-mail: lei.xue@ tongji.edu.cn

or T Xu, Howard Hughes Medical Institute, Yale University, 295 Congress Avenue, New Haven, CT 06519, USA. Tel: +1 (203) 737-2623; Fax: +1 (203) 737-1762; E-mail: tian.xu@yale.edu

${ }^{4}$ These authors contribute equally.

Keywords: bendless; cell death; cell migration; JNK; TNF

Abbreviations: TNF, tumor necrosis factor; JNK, c-Jun N-terminal Kinase; MAPK, mitogen-activated protein kinases; GMR, glass multiple reporter; MF, morphogenetic furrow; MARCM, mosaic analysis with a repressible cell marker; ey, eyeless; VNC, ventral nerve cord; MMP1, matrix metalloproteinase 1; A/P, anterior/posterior Received 20.7.13; revised 15.9.13; accepted 25.9.13; Edited by H Steller; published online 25.10.13
} 

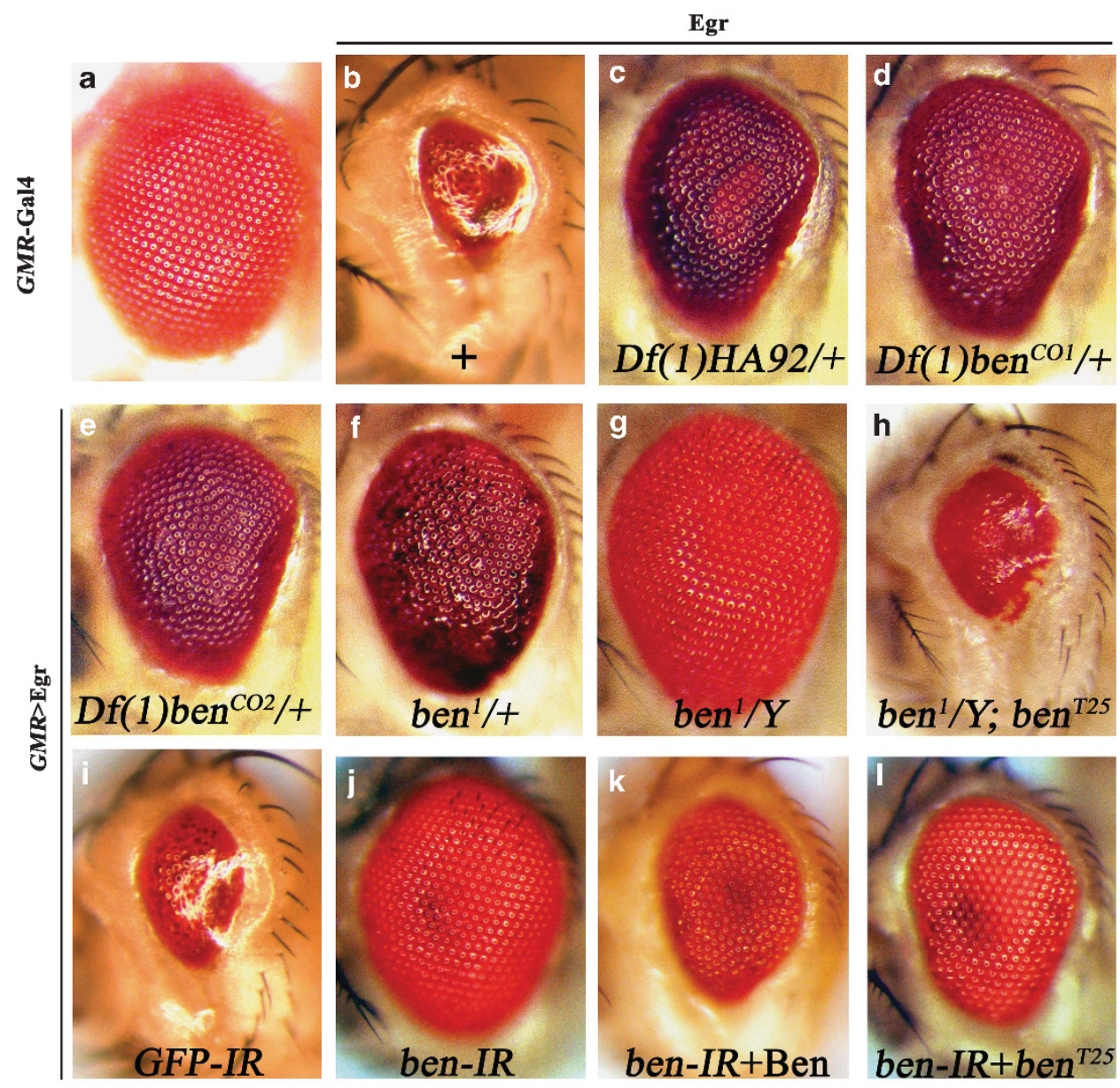

m EP1651

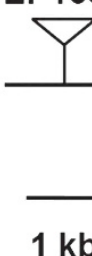

d09936

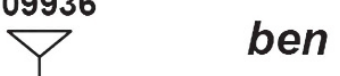

$1 \mathrm{~kb}$

Figure 1 Ben regulates Egr-induced cell death in Drosophila. (a-I) Light micrographs of Drosophila adult eyes are shown. Compared with the controls (a, GMR-Gal4/ + ), GMR $>$ Egr triggers cell death and produces a small eye phenotype (b, UAS-Egr/ +; GMR-Gal4/ +). This phenotype is suppressed partially by $D f(1) H A 92(\mathbf{c}, D f(1) H A 92 / X$; UAS-Egr/ +; GMR-Gal4/+), Df(1)ben ${ }^{C O 1}$ (d, Df(1)ben ${ }^{C O 1} / X ;$ UAS-Egr/ +; GMR-Gal4/+) or Df(1)ben CO2 (e, Df(1)ben $\left.{ }^{C O 2} / X ; U A S-E g r /+; \quad G M R-G a l 4 /+\right)$, or heterozygosity of $\operatorname{ben}^{1}\left(\mathbf{f}\right.$, ben $^{1} / X$; UAS-Egr/ +; GMR-Gal4/ +), and fully by hemizygosity of ben ${ }^{1}\left(\mathbf{g}\right.$, ben $^{1} / Y$; UAS-Egr/ +; GMR-Gal4/ + ) or expression of a ben RNAi (j, UAS-Egr/ +; GMR-Gal4/UAS-ben-IR) but not a GFP RNAi (i, UAS-Egr/UAS-GFP-IR; GMR-Gal4/ + ). The suppression of GMR > Egr eye phenotype can be reversed by ben $^{T 25}$ (h, ben ${ }^{1} / Y$; UAS-Egr/ben ${ }^{T 25} ;$ GMR-Gal4/ben ${ }^{T 25} ;$ I, UAS-Egr/ben ${ }^{T 25} ;$ GMR-Gal4/UAS-ben-IR) or expression of Ben (k, UAS-Egr/UAS-Ben; GMR-Gal4/UAS-ben-IR). (m) The ben gene, ben ${ }^{T 25}$ transgene and two P-elements, EP1651 and d09936, used for ectopic Ben expression are indicated

uncovering the canonical Egr-JNK pathway components, for example, wengen (wgn, encoding an Egr receptor), ${ }^{14}$ dTRAF2, ${ }^{6}$ dTAK $1,{ }^{25,26}$ hep $^{27}$ and basket (bsk, encoding the Drosophila JNK) ${ }^{28}$ were isolated as suppressors in our screen, indicating that the screen was effective. One of the suppressors was mapped cytologically between 12C6 and 12D3, a region uncovered by three deficiencies, $D f(1) H A 92$, $D f(1) b e n^{C O 1}$ and $D f(1) b e n^{C O 2}$, that partially suppressed the GMR $>$ Egr phenotype (Figures $1 \mathrm{c}-\mathrm{e}$ ). This region contains multiple genes including ben, which is located in 12D2, as predicted by the Drosophila genome project. $^{29}$ Further experiments showed that the GMR>Egr eye phenotype was partially suppressed in heterozygous $b e n^{1} /+$ females (Figure 1f) and fully suppressed in hemizygous $b e n^{1} / Y$ males (Figure 1g), which could be reverted by ben ${ }^{T 25}$ (Figure 1h), a genomic rescue fragment for ben (Figure $1 \mathrm{~m}) .{ }^{18}$ Furthermore, expression of a ben RNAi ${ }^{30,31}$ but not a GFP RNAi strongly suppressed the GMR $>$ Egr eye phenotype (Figures $1 \mathrm{i}$ and $\mathrm{j}$ ). The suppression by ben RNAi was partially released by $b e n^{T 25}$ (Figure 1l), or expression of Ben (Figure 1k), confirming the efficacy and specificity of the ben RNAi. Together, these results indicate that Ben is absolutely required for ectopic Egr-induced cell death in eye development. 

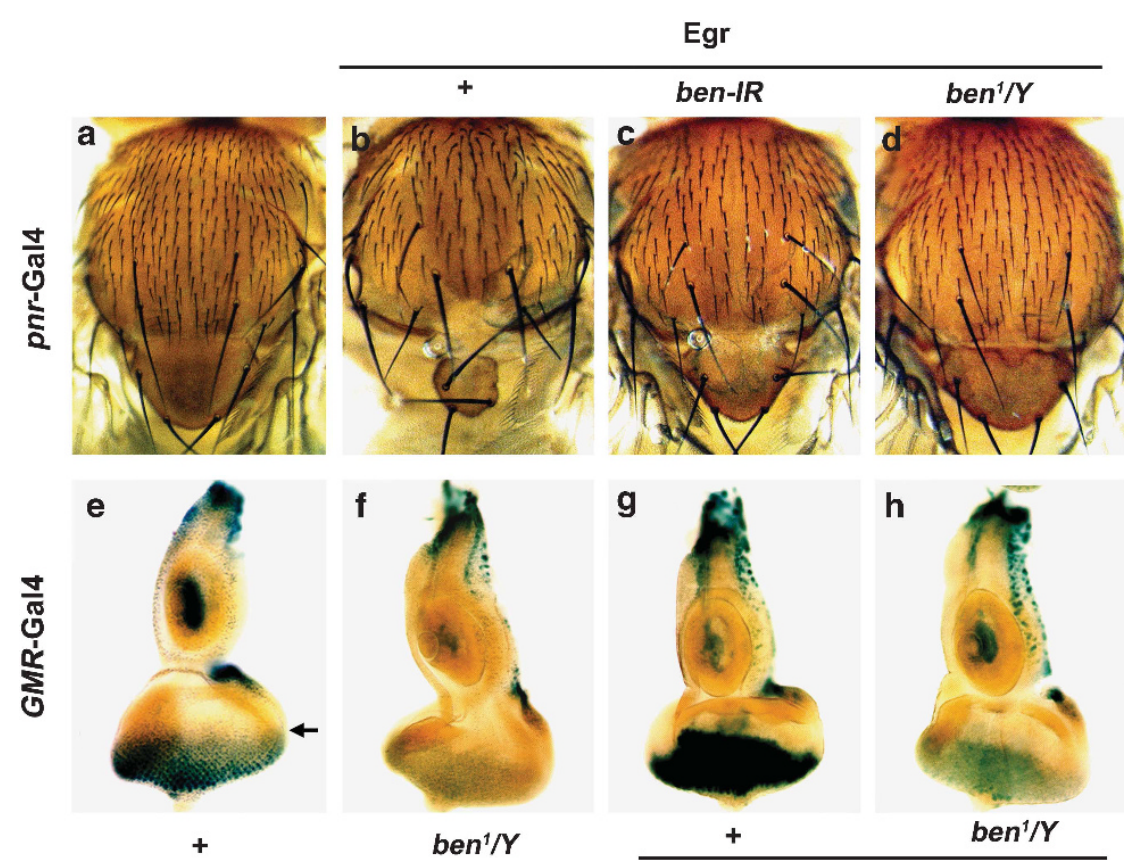

h

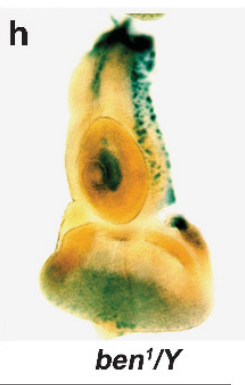

Egr

Figure 2 ben is required for endogenous JNK signaling. (a-d) Light micrographs of Drosophila adult thorax are shown. Compared with wild type (a, pnr-Gal4/ + ), expression of Egr under the control of pnr promoter produces a small scutellum phenotype (b,UAS-Egr/ +; pnr-Gal4/+). The pnr $>$ Egrphenotype is suppressed strongly by a ben RNAi (c, UAS-Egr/ + ; pnr-Gal4/UAS-ben-IR) and fully in ben mutants (d, ben ${ }^{1} / Y$; UAS-Egr/ +; pnr-Gal4/ + ). (e-h) X-Gal staining of 3rd instar larval eye discs is shown. The weak endogenous puc expression is detected after extensive staining (e, GMR-Gal4/puc ${ }^{E 69}$ ). The puc expression posterior to the MF (arrow) is significantly reduced in ben mutants (f, ben $1 / Y$; GMR-Gal4/puc ${ }^{E 69}$ ), whereas the puc expression in disc edge are not affected. GMR>Egr induces puc expression posterior to the morphogenetic furrow (g, UAS-Egr/ +; GMR-Gal4/puc $\left.{ }^{E 69}\right)$, which is strongly suppressed in ben mutants $\left(\mathrm{h}\right.$, ben $^{1} / Y$; UAS-Egr/ +; GMR-Gal4/puc ${ }^{E 69}$ ). Staining was performed at $37^{\circ} \mathrm{C}$ for $12 \mathrm{~h}$ (e and $\left.\mathbf{f}\right)$ or at room temperature for $2 \mathrm{~h}(\mathbf{g}$ and $\mathbf{h})$

To examine the role of Ben in Egr-induced cell death in another developmental context, we characterized the genetic interaction between Ben and Egr in the developing thorax. Ectopic Egr expression in the dorsal thorax driven by pannierGal4 (pnr-Gal4) induced JNK-dependent cell death and produced a small scutellum phenotype (Figure $2 b)^{3,24}$ This phenotype was suppressed by RNAi downregulation (Figure 2c) or mutation (Figure 2d) of ben, suggesting Ben is generally required for ectopic Egr-induced cell death in Drosophila development.

ben is required for endogenous JNK signaling. To investigate the physiological role of ben in modulating JNK signaling, we examined puc-LacZ expression, an in vivo readout of JNK activity, ${ }^{32}$ in third-instar eye discs. Previous study has shown that puc expression posterior to the morphogenetic furrow (MF, arrow in Figure 2e) depends on endogenous Egr function. ${ }^{3}$ We found that such a expression pattern was significantly reduced in ben mutants (Figure 2f). In contrast, puc expression in the disc margin, which is independent of the Egr signaling, ${ }^{3}$ remained unaffected (Figures $2 e$ and $f$ ). Besides, GMR $>$ Egr sturdily activated puc transcription posterior to the MF (Figure $2 \mathrm{~g}$ ), ${ }^{3}$ which was considerably suppressed by loss of ben (Figure $2 \mathrm{~h}$ ). Together, these data suggest that Ben is physiologically required for both endogenous and ectopic Egr-induced JNK activation.

Ben regulates JNK signaling through dTRAF2. To genetically map Ben in the Egr-JNK pathway, we examined the genetic interaction between Ben and dTAK1 or Hep in the developing eyes. Expression of dTAK1 or a constitutive active form of Hep $\left(\mathrm{Hep}^{\mathrm{CA}}\right)$ under the control of the sevenless (sev) promoter (sev $>$ dTAK1 and sev $>\mathrm{Hep}^{\mathrm{CA}}$ ) trigged extensive cell death in larval eye discs and produced rough eyes with reduced sizes (Figures $3 a$ and $c$ ). ${ }^{6,25,26}$ These phenotypes were suppressed by reducing bsk expression ${ }^{23}$ but remained unaffected in ben mutants (Figures $3 b$ and $d$ ), suggesting that Ben acts upstream of dTAK1 and Hep in the Egr-JNK signaling pathway.

Consistent with its role in regulating JNK signaling, ectopic expression of Ben in developing eyes produced small and rough eyes (Figure $3 e$ ), which were suppressed by loss of dTRAF2 (Figure $3 \mathrm{~g}$ ) or dTAK1 (Figure $3 \mathrm{~h}$ ) but not that of wgn (Figure 3f). Furthermore, Ben expression in the dorsal thorax driven by pnr-GAL4 resulted in a small scutellum phenotype (Figure $3 \mathrm{i}$ ), which was also suppressed by loss of dTRAF2 (Figure 3k) or dTAK1 (Figure 3l) but not that of wgn (Figure 3j). Collectively, these data indicate that Ben acts upstream of dTRAF2 and downstream of Wgn in modulating JNK signaling.

Ben physically binds dTRAF2 and promotes dTRAF2 polyubiquitination. Ben encodes an E2 ubiquitin-conjugating enzyme that has been conserved from yeast to mammals. ${ }^{33,34}$ Our genetic data suggested that Ben acts upstream of dTRAF2, which encodes a RING finger-containing E3 ubiquitin-ligating enzyme. To test whether Ben could physically interact with dTRAF2, we examined the 

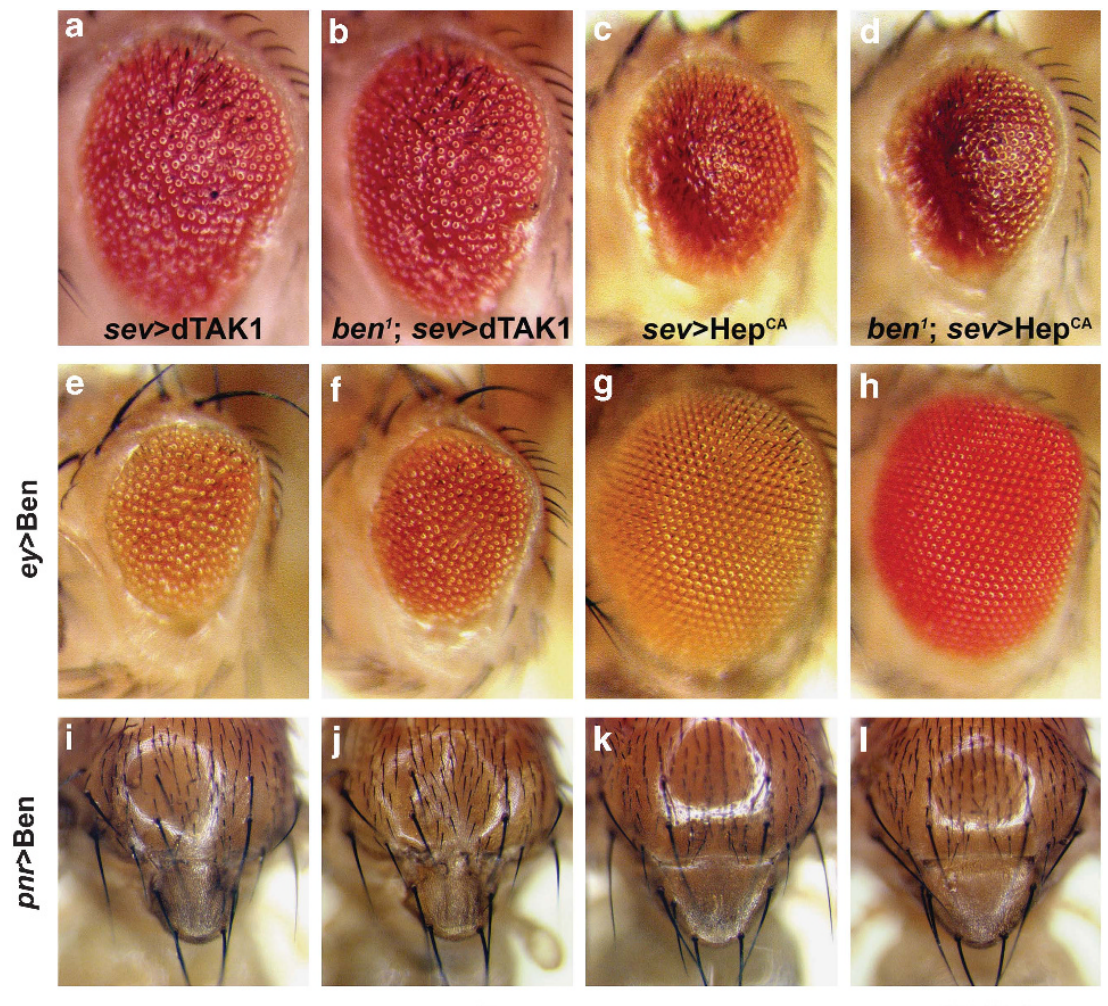

Figure 3 Ben regulates JNK signaling through dTRAF2. Genetic epistatic analysis of Ben in Egr-JNK pathway. (a-h) Light micrographs of Drosophila adult eyes are shown. Expression of dTAK1 (a, sev-Gal4 UAS-dTAK1/ +) or a constitutive active form of Hep (c, sev-Gal4 UAS-HepCA + ) in developing eyes under the control of sev promoter induces apoptosis and generates rough eyes with reduced size. These phenotypes are not suppressed in ben mutants (b, ben ${ }^{1}$; sev-Gal 4 UAS-dTAK1/ + and $\mathbf{d}$, ben $^{1}$; sev-Gal4 UAS-Hep ${ }^{\mathrm{CA}} /+$ ). Expression of Ben in developing eyes under the control of ey promoter induces apoptosis and generates rough eyes with reduced size (e, ben $^{d 09936} / \mathrm{Y} ;$ ey-Gal4/ + ). This phenotype is suppressed in dTRAF2 mutants ( $g, d T R A F 2^{E X 1}$ ben $^{d 09936} / \mathrm{Y} ;$ ey-Gal4/ + ) or by the expression of a dTAK1 RNAi (h, ben ${ }^{d 09936} / \mathrm{Y}$; ey-Gal4/UAS-dTAK1-IR) but not by that of wgn (f, ben ${ }^{d 09936} / Y$; ey-Gal4/UAS-Wgn-IR). (i-l) Light micrographs of Drosophila adult thorax are shown. Expression of Ben under the control of pnr promoter produces a small scutellum phenotype $\left(\mathbf{i}, b^{E} n^{E P 1651} / \mathrm{Y} ;\right.$ pnr-Gal4/ + ). The pnr $>$ Ben phenotype is suppressed in $d T R A F 2$ mutants $\left(\mathbf{k}, d T R A F 2{ }^{E X 1}\right.$

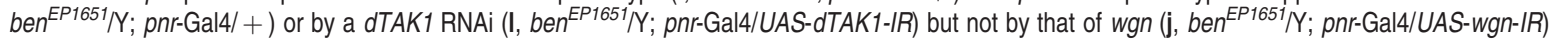

subcellular localization of Ben and dTRAF2 in the developing eyes. Immunostaining of third-instar eye discs showed colocalization of Ben and dTRAF2 on the cell membrane posterior to MF (Figures 4a-f). In addition, co-immunoprecipitation experiment confirmed that ectopically expressed Flag-dTRAF2 could physically interact with endogenous (Figure 4g, lane 2) or ectopically expressed Ben (Figure $4 \mathrm{~g}$, lane 3 ) in vivo. As a negative control, the endogenous Ben protein could not be precipitated by antiFlag M2 affinity gel in the absence of Flag-dTRAF2 (Figure 4g, lane 1). Parkin (Park) encodes another RING finger-containing E3 ubiquitin-ligating enzyme. We found that Flag-tagged Park (Park-Flag) failed to precipitated endogenous (Figure 4h, lane 2) or ectopically expressed Ben (Figure 4h, lane 3), further confirming that the physical interaction between Ben and dTRAF2 is specific. Previous studies have shown that TNF signaling induces K63-linked polyubiquitination of TRAF2/6. ${ }^{35}$ This polyubiquitination does not target TRAF proteins for proteasome-dependent degradation, but instead serves as a signal to activate downstream pathway. ${ }^{36}$ In agreement with the mammalian data, we found that ectopic Egr expression stimulated dTRAF2 polyubiquitination (Figure $4 \mathrm{i}$, lane 3 , upper) but did not affect the stability of dTRAF2 protein (Figure 4i, lane 3, middle). Loss of
Ben blocked Egr-induced polyubiquitination of dTRAF2 (Figure 4i, lane 4), whereas ectopic Ben expression induced dTRAF2 polyubiquitination without affecting its protein stability (Figure $4 \mathrm{i}$, land 5 ). Thus, Ben is both necessary and sufficient for Egr-induced polyubiquitination of dTRAF2.

ben regulates JNK-dependent oxidative stress resistance and lifespan. The JNK signaling pathway has been implicated in regulating the oxidative stress resistance and normal lifespan in Drosophila. ${ }^{5,6}$ Consistent with its role in modulating JNK signaling, ben mutants displayed a significantly compromised resistance against treatment of paraquat (Figure 5a), an herbicide that induces the formation of reactive oxygen species, ${ }^{37}$ and shortened mean and maximal lifespan (Figure $5 b$ ). Both defects were rescued by $b^{b e n^{T 25}}$ (Figures 5a and b), confirming that loss of ben function is responsible for the reduced oxidative stress resistance and shortened lifespan in ben mutants. We found that expression of the JNK kinase Hep in the nervous system (elav > Hep) fully rescued the compromised oxidative stress tolerance defect and shortened lifespan in ben mutants (Figures $5 \mathrm{a}$ and $\mathrm{b}$ ), suggesting that Ben modulates these physiological functions through JNK signaling. 

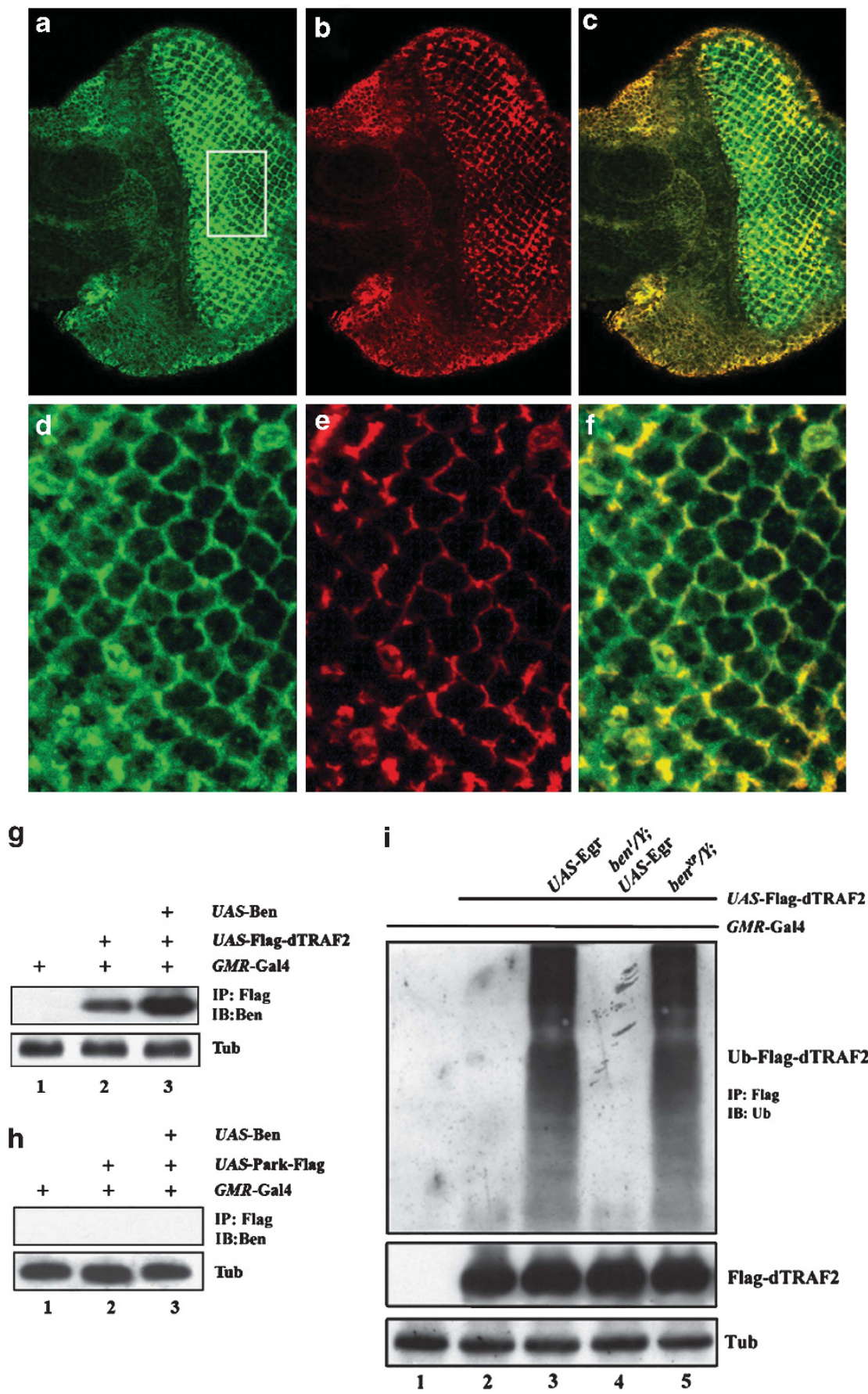

Figure 4 Ben binds to dTRAF2 and regulates dTRAF2 polyubiquitination. (a-f) Confocal images of Drosophila 3rd instar eye discs are shown. Ben (a and d) and dTRAF2 (b and $\mathbf{e}$ ) co-localized to the cell membrane. (c and f) are merged pictures. (d-f) are high magnification of a-c (boxed area in $\mathbf{a})$. (g) Ben physically interacts with Flag-dTRAF2 in vivo. Endogenous (lane 2, GMR-Gal4 UAS-Flag-dTRAF2/ + ) and ectopically expressed (lane 3, UAS-Ben; GMR-Gal4 UAS-Flag-dTRAF2/ + ) Ben binds to Flag-dTRAF2 driven by GMR-Gal4 in immunoprecipitation assay. GMR-Gal4 alone served as the negative control (lane 1, GMR-Gal4/ +). IP, anti-Flag; IB detection, anti-Ben. $\beta$-tubulin served as a loading control (bottom). (h) Ben fails to interact with Park-Flag in vivo. Endogenous (lane 2, GMR-Gal4 UAS-Park-Flag/ +) and ectopically expressed (lane 3 , UAS-Ben; GMR-Gal4 UAS-Park-Flag/ + ) Ben does not bind to Park-Flag driven by GMR-Gal4 in immunoprecipitation assay. GMR-Gal4 alone served as the negative control (lane1, GMR-Gal4/ + ). IP, anti-Flag; IB detection, anti-Ben. $\beta$-tubulin served as a loading control (bottom). (i). The expression (middle) and polyubiquitination of Flag-dTRAF2 driven by GMR-Gal4. While dTRAF2 protein level remains constant (lane 2-5), the weak polyubiquitination of dTRAF2 (lane 2, GMR-Gal4 UAS-Flag-dTRAF2/ +) is significantly enhanced by the expression of Egr (lane 3, UAS-Egrl + ; GMR-Gal4 UAS-Flag-dTRAF2/ + ) or Ben (lane 5, ben ${ }^{\text {dog936 }}$; GMR-Gal4 UAS-Flag-dTRAF2/ + ). Loss of ben blocks the Egr- induced dTRAF2 polyubiquitination (lane 4, ben ${ }^{1}$; UAS-Egr/ + ; GMR-Gal4 UAS-Flag-dTRAF2/ + ). GMR-Gal4 alone serves as a negative control (lane 1, GMR-Gal4/ + ). $\beta$-tubulin was used as a loading control (bottom). Data are representative of three independent experiments 

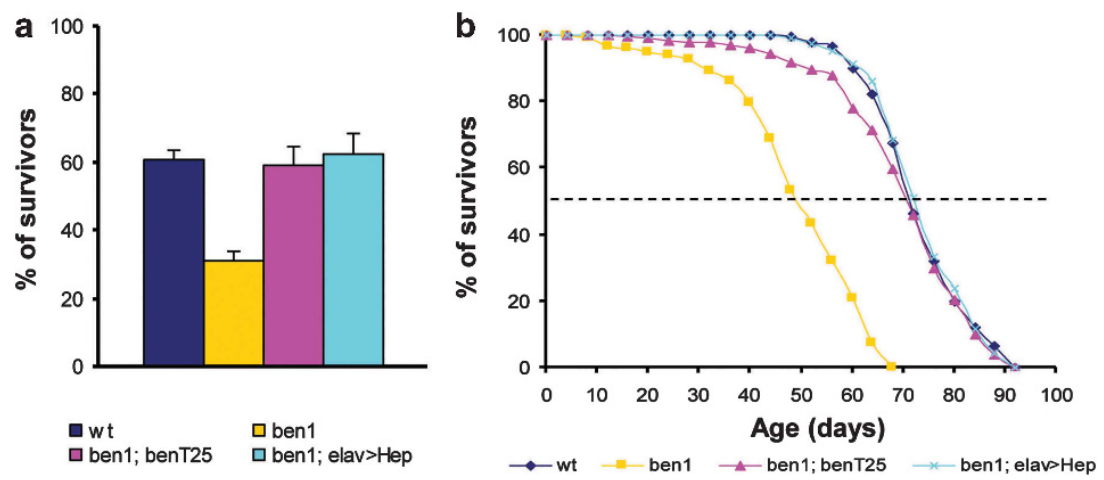

Figure 5 ben regulates JNK-dependent oxidative stress resistance and lifespan in Drosophila. (a) Loss of ben compromises oxidative stress resistance. Three-day-old flies were fed with $15 \mathrm{mM}$ paraquat in $5 \%$ sucrose for $24 \mathrm{~h}$, and their survival rates were measured. ben mutant males (ben1: $\mathrm{w}^{1118}$ ben $^{1} / \mathrm{Y}$ ) showed significant reduction in survival rate as compared with wild-type males (wt: $\mathrm{w}^{1118} / \mathrm{Y}$ ). This reduction was fully rescued by ben ${ }^{T 25}$ (ben1; benT25: $\mathrm{w}^{1118}$ ben $^{1} / Y$; ben ${ }^{T 25}$ ) or neural expression of Hep (ben1; elav > Hep: $\mathrm{w}^{1118}$ ben $^{1} / Y$; UAS-Hep ${ }^{\mathrm{WT}} /+$; elav-Gal4/ +). (b) Loss of ben reduces lifespan. ben mutant males show significant reduction of median and maximal lifespan as compared with wild-type males. This reduction could be rescued by ben ${ }^{T 25}$ or neural expression of Hep. Survival of three independent cohorts was monitored over time. Cohort sizes and $P$-values are as follows: wt, $n=197$; ben1, $n=230$; ben1; benT25, $n=295$; ben1; elav $>$ Hep, $n=260 ; P<0.01$

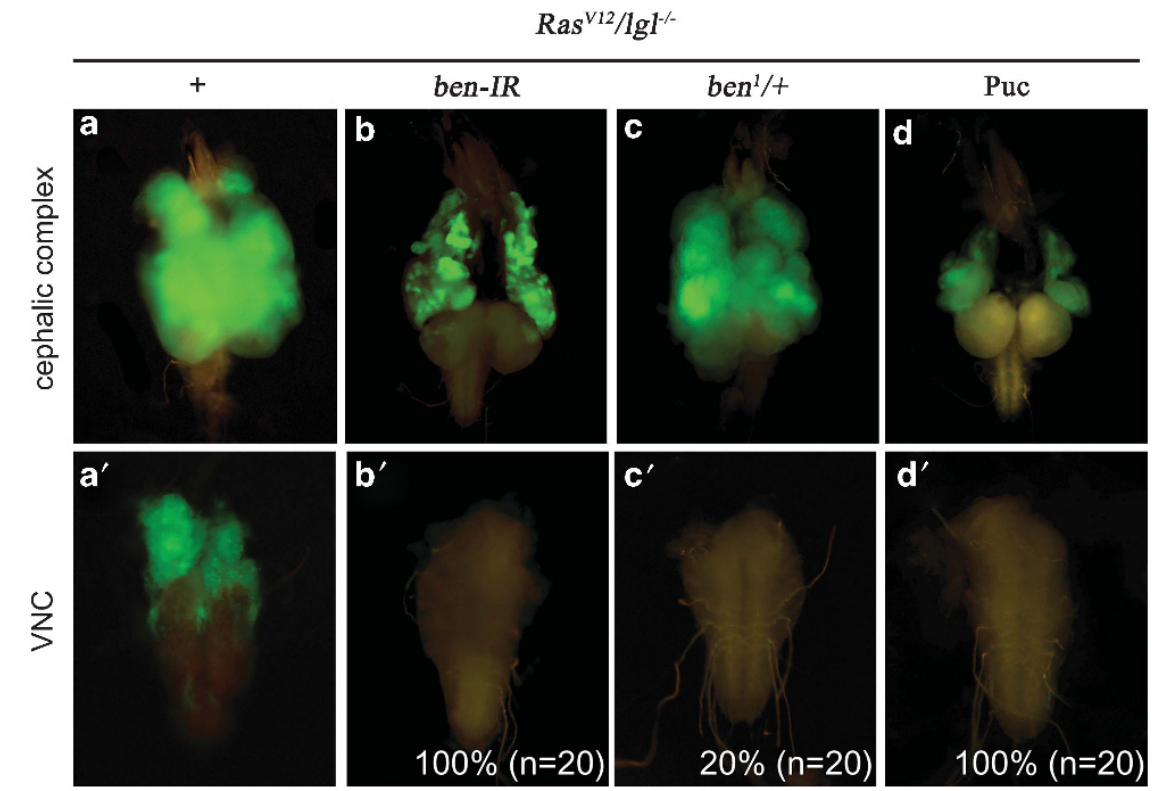

Figure 6 ben is required for Ras-lgl induced tumor growth and invasion. GFP-labeled clones of cells with indicated genotypes were created in developing eye-antennal discs. Ras ${ }^{V 12} /|g|^{-1-}$ induced tumor growth (a) and invasion to the VNC (a') were suppressed by loss of ben (b and $\mathbf{c}$ ) or expressing of Puc (d). (a) $y, w$, ey-Flp/ $+;$ tub-Gal80,

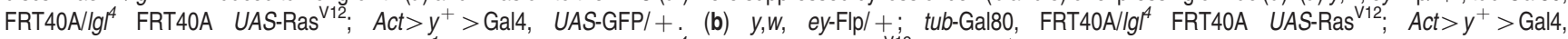

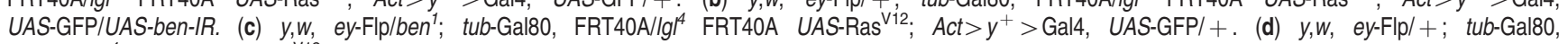
FRT40A $/$ II $^{4}$ FRT 40A UAS-Ras ${ }^{\mathrm{V} 12} ;$ Act $>y^{+}>$Gal4, UAS-GFP/UAS-PuC

ben is required for Ras-Igl-induced tumor growth and invasion. As previously reported, expression of oncogenic Ras in $I g l$ mutant cells ( $\operatorname{Ras}^{V 12} / \mid g^{-/-}$) in eye-antennal discs using the ey-FLP/MARCM system resulted in tumor-like growth (Figure 6a) with invasive migration into the ventral nerve cord (VNC) of the central nerve system (Figure $\left.6 a^{\prime}\right)^{7,9,13,38}$ The Egr-JNK signaling has been implicated as an important mediator of this oncogenic cooperation-induced tumor progression. ${ }^{7,8}$ Consistently, we found that inactivation of JNK signaling by expression of the JNK phosphatase Puc could dramatically suppress the growth and invasion behavior of $\operatorname{Ras}^{V 12} / \mid g^{-/-}$tumors (Figures $6 d-d^{\prime}$ ).

To investigate whether ben is also involved in $\operatorname{Ras}^{V 12} / \mid g^{I^{-}}$ -triggered tumor progression, we knocked down ben expression in tumor cells by expressing the ben RNAi or introduced the $\operatorname{Ras}^{V 12} / \mid \mathrm{gl}^{-/-}$tumor into heterozygous ben ${ }^{1}$ mutants. We found that RNAi downregulation of ben phenocopied inactivation of JNK signaling, in which both tumor growth (Figure 6b) and invasion to the VNC (Figure $6 b^{\prime}$ ) were significantly suppressed. Consistent with a weak suppression of the GMR>Egr eye phenotype (Figure 1f), 

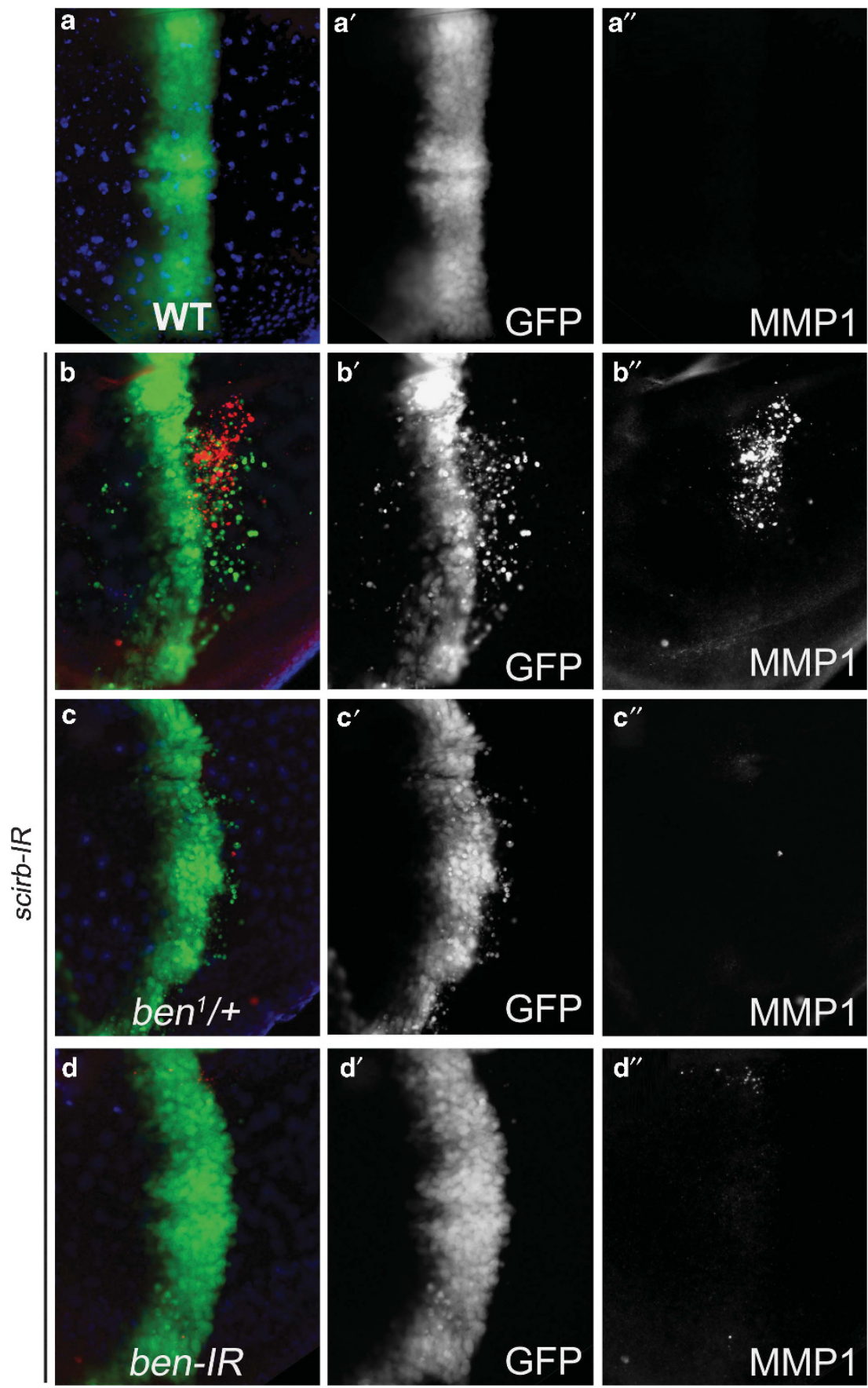

Figure 7 ben is required for JNK-mediated cell migration. (a-d) Fluorescence micrographs of wing discs are shown. Compared with the controls (a and $\mathbf{a}^{\prime \prime}$, ptc-Gal4 UAS-GFP/ +), RNAi downregulation of scrib (b, ptc-Gal4 UAS-GFP/UAS-scrib-IR) induced cell migration ( $\left.\mathbf{b}^{\prime}\right)$ and MMP1 upregulation ( $\left.\mathbf{b}^{\prime \prime}\right)$ were suppressed by mutation (c, ben $^{1} /+$; ptc-Gal4 UAS-GFP/UAS-scrib-IR) or RNAi downregulation (d, ptc-Gal4 UAS-GFP/UAS-scrib-IR; UAS-ben-IR/ +) of ben

heterozygosis of $b e n^{1}$ mutation partially suppressed the tumor growth and invasion phenotype (Figures $\left.6 c-c^{\prime}\right)$. Thus, ben is required for $\operatorname{Ras}^{V 12} / \mid \mathrm{gl}^{-/-}$triggered JNK signaling-mediated tumor growth and invasion.

ben is required for JNK-dependent cell invasion and MMP1 expression. The epithelia of the Drosophila wing imaginal disc have been established as another in vivo model to study invasive cell migration. ${ }^{10,39,40}$ It was shown that downregulation of cell polarity genes, for example, disc large $(d / g)$, resulted in JNK-dependent cell invasion and upregulation of $\mathrm{MMP}_{1}{ }^{8}$ a matrix metalloprotease essential for basement membrane degradation. ${ }^{38,41,42}$ Consistently, RNAi downregulation of the cell polarity gene scrib driven by ptc-Gal4 along the anterior/posterior (A/P) compartment boundary of wing imaginal discs (Figures $7 \mathrm{a}-\mathrm{a}^{\prime \prime}$ ) resulted in a JNK-dependent invasion-like phenotype, ${ }^{8}$ in which cells delaminated and migrated away from the A/P boundary, 
accompanied with increased expression of MMP1 (Figures $7 b-b^{\prime \prime}$ ). We found both the cell migration phenotype and MMP1 elevation were suppressed by mutation or RNAi downregulation of ben (Figures $7 c-c^{\prime \prime}$ and $d-d^{\prime \prime}$ ), confirming that ben is required for JNK-dependent cell invasion and MMP1 expression induced by loss of cell polarity gene.

\section{Conclusions}

We have identified Ben as a crucial regulator of JNKdependent cell death and invasion, tumor progression, oxidative stress resistance and longevity in Drosophila. Our genetic epistasis and biochemical analysis established Ben as a novel component of the Egr-JNK signaling pathway via acting upstream of dTRAF2. Given the evolutionary conservation of the JNK pathway, the ortholog of Ben might have a similar role in modulating JNK-mediated cell death, tumor progression, stress response and longevity in other animals.

\section{Materials and Methods \\ Drosophila strains and generation of clones. Fluorescently labeled invasive tumors were produced in the eye discs as previously described ${ }^{13}$ using the following strains: $y, w$, ey-Flp; tub-Gal80, FRT40A; Act $>y^{+}>$Gal4, UAS-GFP (40A tester) and $|g|^{4}$ FRT40A UAS-Ras ${ }^{12}$ (40A tester). Additional strains including elav-Gal4, GMR-Gal4, sev-Gal4, ey-Gal4, pnr-Gal4 drivers and ben ${ }^{E P 1651}$, UAS-GFP-IR were obtained from Bloomington stock center; ben ${ }^{\text {do9936 }}$ was obtained from Harvard Exelixis collection; ben ${ }^{143}$ and ben ${ }^{\text {T25 }}{ }_{18}$ UAS-Ben, ${ }^{19}$ UAS-ben-IR ${ }^{30,31} ;$ DTRAF2 ${ }^{\text {ex1 }}$ and hep ${ }^{1,27}$ bsk $^{1,28}{ }^{28}$ puc ${ }^{E 69},{ }^{44}$ UAS-Egr, ${ }^{3}$ UAS-dTAK1, ${ }^{26}$ UAS-Hep ${ }^{\text {CA }}$, UAS-Wgn-IR, ${ }^{14}$ UAS-scrib-IR, UAS-dTAK1-IR, ${ }^{24}$ UAS-dTRAF2-IR, UAS-Puc and UAS-Flag-dTRAF2 ${ }^{6}$ were previously described.}

Immunohistochemistry. Antibody staining of wing and eye imaginal discs was performed as previously described. ${ }^{24} \mathrm{X}$-gal staining of eye discs was performed as described. ${ }^{45}$

Lifespan and oxidative stress resistance assay. Stocks were backcrossed with $w^{1118}$ for more than eight generations, and lifespan and oxidative stress resistance were monitored at $25^{\circ} \mathrm{C}$ as previously described. ${ }^{5}$

Immunoprecipitation and western blot. Heads were cut from freshly eclosed flies of indicated genotypes and homogenized in lysis buffer. ${ }^{46}$ Immunoprecipitation, western blot, antibody staining and detection were performed as previously described. ${ }^{46}$

\section{Conflict of Interest}

The authors declare no conflict of interest.

Acknowledgements. We thank R Wyman, M Tanouye, J Chung, M Miura, $\mathrm{K}$ Basler, D Bohmann, Bloomington, Harvard, VDRC and NIG stock centers for fly stocks, A Srivastava for technical assistance and M Ho for discussion and comments. This work was supported by the National Basic Research Program of China (973 Program) (grant no. 2010CB944901 and 2011CB943903), National Natural Science Foundation of China (grant no. 31071294, 31171413 and 31371490), the PhD Programs Foundation of Ministry of Education of China (grant no. 20120072120030 and 20120072110023) and Shanghai Committee of Science and Technology (grant no. 09DZ2260100) to LX, the National Institutes of Health grant to TX. TX is an investigator of the Howard Hughes Medical Institute.

1. Wajant $\mathrm{H}$, Pfizenmaier $\mathrm{K}$, Scheurich $\mathrm{P}$. Tumor necrosis factor signaling. Cell Death Differ 2003; 10(1): 45-65.

2. Davis RJ. Signal transduction by the JNK group of MAP kinases. Cell 2000; 103(2): 239-252.
3. Igaki T, Kanda H, Yamamoto-Goto Y, Kanuka H, Kuranaga E, Aigaki T et al. Eiger, a TNF superfamily ligand that triggers the Drosophila JNK pathway. EMBO J 2002; 21(12): 3009-3018.

4. Moreno E, Yan M, Basler K. Evolution of TNF signaling mechanisms: JNK-dependent apoptosis triggered by Eiger, the Drosophila homolog of the TNF superfamily. Curr Biol 2002; 12(14): 1263-1268.

5. Wang MC, Bohmann D, Jasper $\mathrm{H}$. JNK signaling confers tolerance to oxidative stress and extends lifespan in Drosophila. Dev Cell 2003; 5(5): 811-816.

6. Xue L, Igaki T, Kuranaga E, Kanda H, Miura M, Xu T. Tumor suppressor CYLD regulates JNK-induced cell death in Drosophila. Dev Cell 2007; 13(3): 446-454.

7. Igaki T, Pagliarini RA, Xu T. Loss of cell polarity drives tumor growth and invasion through JNK activation in Drosophila. Curr Biol 2006; 16(11): 1139-1146.

8. Cordero JB, Macagno JP, Stefanatos RK, Strathdee KE, Cagan RL, Vidal M. Oncogenic Ras diverts a host TNF tumor suppressor activity into tumor promoter. Dev Cell 2010; 18(6): 999-1011.

9. Brumby AM, Richardson HE. scribble mutants cooperate with oncogenic Ras or Notch to cause neoplastic overgrowth in Drosophila. EMBO J 2003; 22(21): 5769-5779.

10. Vidal M, Larson DE, Cagan RL. Csk-deficient boundary cells are eliminated from normal Drosophila epithelia by exclusion, migration, and apoptosis. Dev Cell 2006; 10(1): 33-44.

11. Vidal M, Warner S, Read R, Cagan RL. Differing Src signaling levels have distinct outcomes in Drosophila. Cancer Res 2007; 67(21): 10278-10285.

12. Doggett K, Grusche FA, Richardson HE, Brumby AM. Loss of the Drosophila cell polarity regulator Scribbled promotes epithelial tissue overgrowth and cooperation with oncogenic Ras-Raf through impaired Hippo pathway signaling. BMC Dev Biol 2011; 11: 57.

13. Pagliarini RA, Xu T. A genetic screen in Drosophila for metastatic behavior. Science 2003 . 302(5648): 1227-1231.

14. Kanda H, Igaki T, Kanuka H, Yagi T, Miura M. Wengen, a member of the Drosophila tumor necrosis factor receptor superfamily, is required for Eiger signaling. J Biol Chem 2002; 277(32): 28372-28375.

15. Geuking P, Narasimamurthy R, Basler K. A genetic screen targeting the tumor necrosis factor/Eiger signaling pathway: identification of Drosophila TAB2 as a functionally conserved component. Genetics 2005; 171(4): 1683-1694.

16. Thomas JB, Wyman RJ. A mutation in Drosophila alters normal connectivity between two identified neurones. Nature 1982; 298(5875): 650-651.

17. Muralidhar MG, Thomas JB. The Drosophila bendless gene encodes a neural protein related to ubiquitin-conjugating enzymes. Neuron 1993; 11(2): 253-266.

18. Oh CE, McMahon R, Benzer S, Tanouye MA. bendless, a Drosophila gene affecting neuronal connectivity, encodes a ubiquitin-conjugating enzyme homolog. J Neurosci 1994; 14(5 Pt 2): 3166-3179.

19. Uthaman SB, Godenschwege TA, Murphey RK. A mechanism distinct from highwire for the Drosophila ubiquitin conjugase bendless in synaptic growth and maturation. $J$ Neurosci 2008; 28(34): 8615-8623.

20. Zhao H, Zheng $X$, Yuan $X$, Wang $L$, Wang $X$, Zhong $Y$ et al. ben Functions with scamp during synaptic transmission and long-term memory formation in Drosophila. $J$ Neurosci 2009; 29(2): 414-424.

21. Zhou R, Silverman N, Hong M, Liao DS, Chung Y, Chen ZJ et al. The role of ubiquitination in Drosophila innate immunity. J Biol Chem 2005; 280(40): 34048-34055.

22. Merkle JA, Rickmyre JL, Garg A, Loggins EB, Jodoin JN, Lee E et al. no poles encodes a predicted E3 ubiquitin ligase required for early embryonic development of Drosophila. Development 2009; 136(3): 449-459.

23. Ma X, Huang J, Yang L, Yang Y, Li W, Xue L. NOPO modulates Egr-induced JNKindependent cell death in Drosophila. Cell Res 2012; 22(2): 425-431.

24. Ma X, Yang L, Yang Y, Li M, Li W, Xue L. dUev1a modulates TNF-JNK mediated tumor progression and cell death in Drosophila. Dev Biol 2013; 380(2): 211-221.

25. Takatsu Y, Nakamura M, Stapleton M, Danos MC, Matsumoto K, O'Connor MB et al. TAK1 participates in C-Jun N-terminal kinase signaling during Drosophila development. Mol Cell Biol 2000; 20(9): 3015-3026.

26. Mihaly J, Kockel L, Gaengel K, Weber U, Bohmann D, Mlodzik M. The role of the Drosophila TAK homologue dTAK during development. Mech Dev 2001; 102(1-2): 67-79.

27. Glise B, Bourbon H, Noselli S. hemipterous encodes a novel Drosophila MAP kinase kinase, required for epithelial cell sheet movement. Cell 1995; 83(3): 451-461.

28. Riesgo-Escovar JR, Jenni M, Fritz A, Hafen E. The Drosophila Jun-N-terminal kinase is required for cell morphogenesis but not for DJun-dependent cell fate specification in the eye. Genes Dev 1996; 10(21): 2759-2768.

29. Adams MD, Celniker SE, Holt RA, Evans CA, Gocayne JD, Amanatides PG et al. The genome sequence of Drosophila melanogaster. Science 2000; 287(5461): 2185-2195.

30. Cronin SJ, Nehme NT, Limmer S, Liegeois S, Pospisilik JA, Schramek D et al. Genome-wide RNAi screen identifies genes involved in intestinal pathogenic bacterial infection. Science 2009; 325(5938): 340-343.

31. Mummery-Widmer JL, Yamazaki M, Stoeger T, Novatchkova M, Bhalerao S, Chen D et al. Genome-wide analysis of Notch signalling in Drosophila by transgenic RNAi. Nature 2009; 458(7241): 987-992.

32. Agnes F, Suzanne M, Noselli S. The Drosophila JNK pathway controls the morphogenesis of imaginal discs during metamorphosis. Development 1999; 126(23): 5453-5462.

33. Hofmann RM, Pickart CM. Noncanonical MMS2-encoded ubiquitin-conjugating enzyme functions in assembly of novel polyubiquitin chains for DNA repair. Cell 1999; 96(5): 645-653. 
34. Deng L, Wang C, Spencer E, Yang L, Braun A, You J et al. Activation of the lkappaB kinase complex by TRAF6 requires a dimeric ubiquitin-conjugating enzyme complex and a unique polyubiquitin chain. Cell 2000; 103(2): 351-361.

35. Shi CS, Kehrl JH. Tumor necrosis factor (TNF)-induced germinal center kinase-related (GCKR) and stress-activated protein kinase (SAPK) activation depends upon the E2/E3 complex Ubc13-Uev1A/TNF receptor-associated factor 2 (TRAF2). J Biol Chem 2003; 278(17): 15429-15434

36. Reiley W, Zhang M, Sun SC. Negative regulation of JNK signaling by the tumor suppressor CYLD. J Biol Chem 2004; 279(53): 55161-55167.

37. Ishii N, Takahashi K, Tomita S, Keino T, Honda S, Yoshino K et al. A methyl viologen-sensitive mutant of the nematode Caenorhabditis elegans. Mutat Res 1990 237(3-4): 165-171.

38. Uhlirova M, Bohmann D. JNK- and Fos-regulated Mmp1 expression cooperates with Ras to induce invasive tumors in Drosophila. EMBO J 2006; 25(22): 5294-5304.

39. Portela M, Richardson HE. Death takes a holiday-non-apoptotic role for caspases in cell migration and invasion. EMBO Rep 2013; 14(2): 107-108.
40. Rudrapatna VA, Bangi $E$, Cagan RL. Caspase signalling in the absence of apoptosis drives Jnk-dependent invasion. EMBO Rep 2013; 14(2): 172-177.

41. Beaucher M, Hersperger E, Page-McCaw A, Shearn A. Metastatic ability of Drosophila tumors depends on MMP activity. Dev Biol 2007; 303(2): 625-634.

42. Deryugina El, Quigley JP. Matrix metalloproteinases and tumor metastasis. Cancer Metastasis Rev 2006; 25(1): 9-34.

43. Thomas JB, Wyman RJ. Mutations altering synaptic connectivity between identified neurons in Drosophila. J Neurosci 1984; 4(2): 530-538.

44. Fanto M, Weber U, Strutt DI, Mlodzik M. Nuclear signaling by Rac and Rho GTPases is required in the establishment of epithelial planar polarity in the Drosophila eye. Curr Biol 2000; 10(16): 979-988.

45. Xue L, Noll M. Dual role of the Pax gene paired in accessory gland development of Drosophila. Development 2002; 129(2): 339-346.

46. Kuranaga $\mathrm{E}$, Kanuka $\mathrm{H}$, Igaki $\mathrm{T}$, Sawamoto $\mathrm{K}$, Ichijo $\mathrm{H}$, Okano $\mathrm{H}$ et al. Reaper-mediated inhibition of DIAP1-induced DTRAF1 degradation results in activation of JNK in Drosophila. Nat Cell Biol 2002; 4(9): 705-710. 